MATHEMATICS OF COMPUTATION

Volume 67, Number 223, July 1998, Pages 947-963

S 0025-5718(98)00971-5

\title{
CONVERGENCE ANALYSIS OF A COVOLUME SCHEME FOR MAXWELL'S EQUATIONS IN THREE DIMENSIONS
}

\author{
R. A. NICOLAIDES AND D.-Q. WANG
}

\begin{abstract}
This paper contains error estimates for covolume discretizations of Maxwell's equations in three space dimensions. Several estimates are proved. First, an estimate for a semi-discrete scheme is given. Second, the estimate is extended to cover the classical interlaced time marching technique. Third, some of our unstructured mesh results are specialized to rectangular meshes, both uniform and nonuniform. By means of some additional analysis it is shown that the spatial convergence rate is one order higher than for the unstructured case.
\end{abstract}

\section{Introduction}

Staggered mesh schemes for the numerical solution of Maxwell's equations go back as far as [18]. Over the years this scheme has seen a number of generalizations intended to enhance its usefulness. Mostly, these generalizations are aimed at increasing the geometric complexity that can be handled. Thus, in [3] a tensor formulation was given. This permits the method to be extended from rectangular meshes to meshes defined by curvilinear coordinates. Two dimensional generalizations using quadrilateral meshes are given in [6] and [8].

For three dimensions, hexahedral mesh formulations were proposed in [7] and [9]. These formulations use interpolation to obtain values for nonbasic field components - those which, in the rectangular situation, lie along the primal or dual mesh edges. Another approach is the "control path" method of [4] and [5]. The control path method uses the classical finite difference approach of modifying the finite difference stencil near the boundary of the domain.

A natural generalization of the standard staggered mesh scheme to tetrahedral meshes was given in [10]. A similar technique was used independently in [14] to solve the Navier-Stokes equations. This "covolume" approach does not use any interpolations and is a very natural way to generalize the original rectangular staggered mesh approach. A characteristic feature of covolume schemes is the use of Voronoi-Delaunay mesh pairs to replace the rectangular staggered mesh arrangement.

Very little rigorous analysis of staggered mesh schemes for electromagnetics is available. We know only of the rectangular mesh analysis of [11] and [12]. For the incompressible Navier-Stokes equations results may be found in [14], [15], and

Received by the editor September 15, 1995 and, in revised form, March 25, 1996.

1991 Mathematics Subject Classification. Primary 65N30, 65N15, 35L50.

Key words and phrases. Maxwell's equations, covolume schemes, unstructured meshes, convergence. 
[16]. Our goal in this paper is to provide a rigorous proof of convergence for the covolume discretization applied to the interior problem for Maxwell's equations. The corresponding analysis for the exterior problem involves the application of radiation conditions at a finite distance from the scatterer and will be given in a subsequent report.

The contents of the paper are as follows. In the first section we state Maxwell's equations for bounded domains with perfect conductor boundary conditions. Following that is a section on Voronoi-Delaunay mesh notations and properties. The main result of Section 3 is an error estimate for a semi-discrete covolume approximation to Maxwell's equations. In practice, a kind of leapfrog scheme is used to time march the discrete equations. Section 4 contains an error estimate for the resulting fully discrete approximation to Maxwell's equations. In the last section we specialize our results to the rectangular case. The rate of convergence is shown to be one order higher than for an arbitrary triangulation.

\section{MAXWELl'S EQUATIONS}

Let $\Omega$ be a bounded domain in $R^{3}$ with boundary $\Gamma$ and unit outward normal $\mathbf{n}$. Let the constants $\epsilon$ and $\mu$ denote, respectively, the electric and magnetic permeabilities of the medium occupying $\Omega$. Then if $\mathbf{E}(x, t)$ and $\mathbf{H}(x, t)$ denote, respectively, the electric and magnetic fields, Maxwell's equations [2] are:

$$
\begin{array}{llrr}
\epsilon \mathbf{E}_{t}-\operatorname{curl} \mathbf{H}=\mathbf{J} & \text { in } & & \Omega \times(0, T), \\
\mu \mathbf{H}_{t}+\operatorname{curl} \mathbf{E}=0 & \text { in } & & \Omega \times(0, T), \\
\operatorname{div}(\epsilon \mathbf{E})=\rho & \text { and } & & \operatorname{div}(\mu \mathbf{H})=0,
\end{array}
$$

where $\mathbf{J}=\mathbf{J}(x, t)$ is a known applied current and $\rho(x, t)$ is a charge density. We shall assume a perfect conductor boundary condition so that

$$
\mathbf{E} \times \mathbf{n}=\mathbf{0} \quad \text { on } \quad \Gamma \times(0, T) .
$$

In addition, initial conditions are prescribed so that

$$
\mathbf{E}(x, 0)=\mathbf{E}_{0}(x) \quad \text { and } \quad \mathbf{H}(x, 0)=\mathbf{H}_{0}(x), \quad \forall x \in \Omega,
$$

where $\mathbf{E}_{0}$ and $\mathbf{H}_{0}$ are given functions satisfying

$$
\operatorname{div}\left(\epsilon \mathbf{E}_{0}\right)=\rho(x, 0), \quad \operatorname{div}\left(\mu \mathbf{H}_{0}\right)=0,
$$

and where it is assumed that

$$
\frac{\partial \rho}{\partial t}=\operatorname{div} \mathbf{J}
$$

Let $L^{p}(0, T ; X)$ denote the set of all strongly measurable functions $u(t, \cdot)$ from $[0, T]$ into the Hilbert space $X$ such that

$$
\int_{0}^{T}\|u(t)\|_{X}^{p} d t<\infty \quad 1 \leq p<\infty .
$$

We say $u \in W^{1, p}(0, T ; X)$ if and only if both $u$ and $\frac{\partial u}{\partial t}$ are in $L^{p}(0, T ; X)$. Also $C^{m}(0, T ; X)$ denotes the space of $m$ times continuously differentiable functions from $[0, T]$ into $X$. By $H(\operatorname{curl} ; \Omega)$ and $H(\operatorname{div} ; \Omega)$ we mean the Hilbert spaces defined by

$$
\begin{aligned}
H(\operatorname{curl} ; \Omega): & =\left\{v \in\left(L^{2}(\Omega)\right)^{3}, \operatorname{curl} v \in\left(L^{2}(\Omega)\right)^{3}\right\}, \\
H(\operatorname{div} ; \Omega): & =\left\{v \in\left(L^{2}(\Omega)\right)^{3}, \operatorname{div} v \in L^{2}(\Omega)\right\} .
\end{aligned}
$$


We shall assume the existence of $(\mathbf{E}, \mathbf{H})$ to $(2.1)-(2.5)$ such that

$$
\mathbf{E}, \mathbf{H} \in C^{1}\left(0, T ;\left(L^{2}(\Omega)\right)^{3}\right) \cap C^{0}(0, T ; H(\operatorname{curl} ; \Omega) \cap H(\operatorname{div} ; \Omega)) .
$$

For this, it is sufficient that $\mathbf{J} \in C^{0}\left(0, T ;\left(L^{2}(\Omega)\right)^{3}\right), \rho \in C^{0}\left(0, T ; L^{2}(\Omega)\right)$ and $\frac{\partial \rho}{\partial t} \in$ $L^{2}\left(0, T ; H^{-1}(\Omega)\right)$, see [2].

\section{Mesh notations AND DiscRete VeCtor FIELDS}

In this section some basic properties of dual mesh systems are introduced. These will lead to a detailed formulation and analysis of our numerical schemes in the following sections.

Assume that the polyhedral domain $\Omega$ has a primal family of finite element style tetrahedral partitions, parametrized by the maximum side length which is generically denoted by $h$. We will assume that the ratio of radii of circumscribing spheres and inscribed spheres of all the individual tetrahedra are uniformly bounded above and below as $h$ approaches 0 . A dual mesh is formed by connecting adjacent tetrahedral circumcenters and, in the case of a tetrahedron with a face on a boundary, by connecting their circumcenters with those of their boundary faces. By elementary geometry these dual edges are perpendicular to the associated tetrahedral faces. These connections also form the edges of a set of polyhedra. It follows from elementary geometry that the edges of tetrahedra are perpendicular to and in one-to-one correspondence with the faces of dual polyhedra or "covolumes". The reciprocal orthogonality between edges, and faces is the key to the results which follow.

The $N$ nodes of the tetrahedral mesh are assumed to be numbered sequentially in some convenient way, and likewise the $T$ nodes of dual mesh. Similarly the $F$ faces (edges) and $M$ edges (faces) of the primal (dual) mesh are sequentially numbered. The individual tetrahedra, faces, edges, and nodes of the primal mesh are denoted by $\tau_{i}, \kappa_{j}, \sigma_{k}$, and $\nu_{l}$, respectively. Those of the dual mesh are denoted by primed quantities such as $\sigma_{j}^{\prime}$. A direction is assigned to each primal edge by the rule that the positive direction is from low to high node number. The dual edges are directed by the corresponding rule. We also denote $F_{1}$ the number of tetrahedral interior faces (or dual edges) and $M_{1}$ the number of tetrahedral interior edges (or dual faces). Let $s_{j}$ denote the area of $\kappa_{j}$ and $h^{\prime}{ }_{j}$ the length of $\sigma_{j}^{\prime}$. In $R^{F_{1}}$, where $F_{1}$ denotes the number of interior primal faces, we will introduce the inner product $(\cdot, \cdot)_{W}$ defined by

$$
(u, v)_{W}:=\sum_{\kappa_{j} \in \bar{\Omega}} u_{j} v_{j} s_{j} h_{j}^{\prime}=\left(S u, D^{\prime} v\right)=\left(D^{\prime} u, S v\right)
$$

and denote the resulting inner product space by $\mathcal{U}$ and the associated norm by

$$
\|u\|_{W}:=(u, u)_{W}^{\frac{1}{2}} .
$$

In $(3.1),(\cdot, \cdot)$ denotes the standard Euclidean inner product, $S:=\operatorname{diag}\left(s_{j}\right)$, $D^{\prime}:=\operatorname{diag}\left(h_{j}^{\prime}\right)$ and $W:=S D^{\prime}$ are $F_{1} \times F_{1}$ invertible diagonal matrices. The norm defined by $(3.2)$ is clearly three times a discrete $L^{2}$ norm. Similarly, we will introduce an inner product in $R^{M}$, where $M$ denotes the number of primal edges:

$$
(u, v)_{W^{\prime}}:=\sum_{\kappa_{j}^{\prime} \in \Omega} u_{j} v_{j} s_{j}^{\prime} h_{j}=\left(S^{\prime} u, D v\right)=\left(D u, S^{\prime} v\right)
$$


and denote the inner product space by $\mathcal{U}^{\prime}$. The associated norm is

$$
\|u\|_{W^{\prime}}:=(u, u)_{W^{\prime}}^{\frac{1}{2}}
$$

The notations in(3.3) and (3.4) correspond to those of (3.1) and (3.2). For example, $s^{\prime}{ }_{j}$ denotes the area of dual face $\kappa_{j}^{\prime}$.

For each primal face $\kappa_{i}$ a discrete circulation is defined by

$$
(C u)_{\kappa_{i}}:=\sum_{\sigma_{j} \in \partial \kappa_{i}} u_{j} \tilde{h}_{j}
$$

Similarly, for each interior covolume face $\kappa_{i}^{\prime}$ the discrete circulation is

$$
\left(C^{\prime} u\right)_{\kappa_{i}^{\prime}}:=\sum_{\sigma_{j}^{\prime} \in \partial \kappa_{i}^{\prime}} u_{j} \tilde{h}_{j}^{\prime} .
$$

A tilde on $h_{j}$ or $h_{j}^{\prime}$ means that the quantity is to be taken with a negative sign if the dual edge is directed against the positive sense of the description of $\partial \kappa_{i}$ or $\partial \kappa_{i}^{\prime}$, respectively, and with a positive sign otherwise. The linear operators $C$ and $C^{\prime}$ map from $R^{M}$ to $R^{F_{1}}$ and $R^{F_{1}}$ to $R^{M_{1}}$, respectively.

For each strictly interior dual edge $\sigma_{j}^{\prime}$ we can form a vector whose $i$ th component is the sign of the orientation of the edge relative to the orientation of the $i$ th strictly interior dual face. From these vectors we obtain the $F_{1} \times M_{1}$ matrix $G$ defined as follows:

$$
(G)_{j i}:= \begin{cases}1 & \text { if } \sigma_{j}^{\prime} \text { is oriented positively along } \kappa_{i}^{\prime} \\ -1 & \text { if } \sigma_{j}^{\prime} \text { is oriented negatively along } \kappa_{i}^{\prime} \\ 0 & \text { if } \sigma_{j}^{\prime} \text { does not meet } \kappa_{i}^{\prime} .\end{cases}
$$

Let $w \in R^{M}$ denote the vector whose $k$ th component is the value assigned on the $k$ th primal edge. Denote $w_{1} \in R^{M_{1}}$ the restriction of $w$ to the interior primal edges. So $\left.w\right|_{\Gamma}=0$ means that the components of $w \in R^{M}$ related to the boundary edges are zero. A direct computation shows:

$$
C w=G D w_{1} .
$$

Also it can be verified that if $v \in R^{F_{1}}$,

$$
C^{\prime} v=G^{T} D^{\prime} v
$$

From these two identities we can prove

Lemma 1. With the above definitions of $w, v$ and $w_{1}$ with $\left.w\right|_{\Gamma}=0$,

$$
\left(C w, D^{\prime} v\right)=\left(C^{\prime} v, D w_{1}\right)
$$

Proof. This is proved as follows:

$$
\left(C^{\prime} v, D w_{1}\right)=\left(G^{T} D^{\prime} v, D w_{1}\right)=\left(D^{\prime} v, G D^{\prime} w_{1}\right)=\left(D^{\prime} v, C w\right) .
$$

This lemma provides a discrete analog of the integration formula

$$
\int_{\Omega} \operatorname{curl} \mathbf{E} \cdot \mathbf{H} d x=\int_{\Omega} \operatorname{curl} \mathbf{H} \cdot \mathbf{E} d x
$$

which holds when $\mathbf{E} \times \mathbf{n}=\mathbf{0}$ on $\Gamma$.

For each tetrahedron $\tau_{i}$ a discrete flux is defined by

$$
(\mathcal{D} u)_{i}:=\sum_{\mu_{j} \in \partial \tau_{i}} u_{j} \tilde{s_{j}}, \quad \forall u \in R^{F_{1}} .
$$


By $\tilde{s_{j}}$ we mean $s_{j}$ negatively signed if the corresponding velocity component is directed toward the inside of $\tau_{i}$ and positively signed otherwise.

In addition to $G$ we will introduce another matrix $B_{1}$ of dimension $F_{1} \times T$

$$
\left(B_{1}\right)_{j i}:= \begin{cases}1 & \text { if } \kappa_{j} \text { is oriented positively along } \partial \tau_{i} \\ -1 & \text { if } \kappa_{j} \text { is oriented negatively along } \partial \tau_{i} \\ 0 & \text { if } \kappa_{j} \text { does not meet } \partial \tau_{i} .\end{cases}
$$

It can be checked directly that

$$
\mathcal{D}=B_{1}^{T} S
$$

Using $B_{1}$ we will define the difference operator $\mathcal{P}$ by

$$
\mathcal{P} \phi:=D^{\prime-1} B_{1} \phi, \quad \forall \phi \in R^{T} .
$$

Now we have

\section{Lemma 2.}

$$
B_{1}^{T} C=0
$$

and

$$
(u, \mathcal{P} \phi)_{W}=(\mathcal{D} u, \phi), \quad \forall \phi \in R^{T}, u \in R^{F_{1}} .
$$

Proof. For (3.7) see [17], Theorem 1. For (3.8), we have

$$
(u, \mathcal{P} \phi)_{W}=\left(S D^{\prime} u, D^{\prime-1} B_{1} \phi\right)=\left(B_{1}^{T} S u, \phi\right)=(\mathcal{D} u, \phi) .
$$

We remark that (3.7) and (3.8) provide the discrete analogs of the identity $\operatorname{div}(\operatorname{curl} \mathbf{u})=0$ and Green's formula $\int_{\Omega} \mathbf{u} \cdot \operatorname{grad} \phi d x=\int_{\Omega}(\operatorname{div} \mathbf{u}) \phi d x$ for $\phi \in$ $H_{0}^{1}(\Omega), \mathbf{u} \in H(\operatorname{div} ; \Omega)$, respectively.

For additional details and relations between other discrete operators in VoronoiDelaunay meshes, see [13] and [17].

\section{Semi-Discrete Maxwell's equations}

In this section the covolume method is used to obtain a spatial discretization of Maxwell's equations (2.1)-(2.5) from which we will obtain a formulation of a semidiscrete form of Maxwell's equations. A basic error estimate is given in Theorem 2.

First we introduce, for general field $\mathbf{A}$, its "face averages" $A_{f} \in R^{F}$ and its "edge averages" $A_{e} \in R^{M}$ as follows (refer to Figure 1):

$$
\begin{aligned}
& A_{f}:=\frac{1}{s_{i}} \int_{\kappa_{i}} \mathbf{A} \cdot \mathbf{n} d s, \\
& A_{f}^{\prime}:=\frac{1}{s_{i}} \int_{\kappa_{i}^{\prime}} \mathbf{A} \cdot \mathbf{n} d s, \\
& A_{e}:=\frac{1}{h_{j}} \int_{\sigma_{j}} \mathbf{A} \cdot \mathbf{t} d \sigma, \\
& A_{e}^{\prime}:=\frac{1}{h_{j}^{\prime}} \int_{\sigma_{j}^{\prime}} \mathbf{A} \cdot \mathbf{t} d \sigma,
\end{aligned}
$$




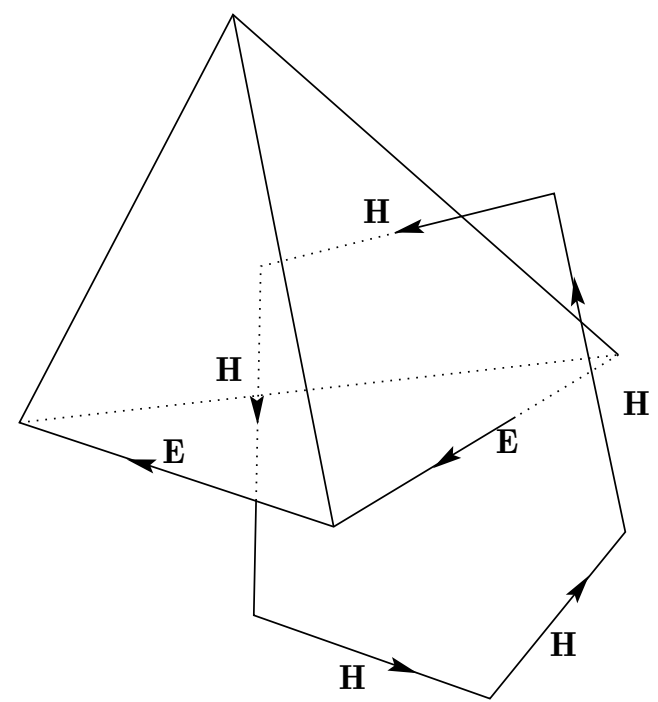

FiguRE 1

where $\mathbf{n}$ and $\mathbf{t}$ denote the unit normal vector to the face $\kappa_{i}$ (or $\kappa_{i}^{\prime}$ ) and the unit tangent vector to the edge $\sigma_{j}$ (or $\sigma_{j}^{\prime}$ ), respectively. Error functions for primal edges and faces will be denoted by

$$
\begin{aligned}
\epsilon_{\mathbf{A}} & :=A-A_{f}, \\
\eta_{\mathbf{A}} & :=A-A_{e}, \\
\delta_{\mathbf{A}} & :=A_{f}-A_{e} .
\end{aligned}
$$

Error functions for dual edges and faces are defined similarly.

As shown in Figure 1, for each tetrahedron $\tau_{i}$ we will use the normal components of the magnetic field $\mathbf{H}$ to its faces and the tangential components of the electric field $\mathbf{E}$ in the directions of its edges. Now integrate both sides of (2.1) over the co-face $\kappa_{j}^{\prime}$ to obtain

$$
\epsilon s_{j}^{\prime} \frac{d\left(E_{f}\right)_{j}}{d t}-\left(C^{\prime} H_{e}\right)_{\kappa_{j}^{\prime}}=\int_{\kappa_{j}^{\prime}} \mathbf{J}(x, t) d x .
$$

Here, $\left(E_{f}\right)_{j}$ denotes the average of $\mathbf{E} \cdot \mathbf{n}_{j}$ over the face $\kappa_{j}^{\prime}$ where the $\mathbf{n}_{j}$ is the unit vector in the direction of $\sigma_{j}$, and $\left(C^{\prime} H_{e}\right)_{\kappa_{j}^{\prime}}$ is the discrete circulation around the face $\kappa_{j}^{\prime}$. Similarly, from $(2.2)$,

$$
\mu s_{i} \frac{d\left(H_{f}\right)_{i}}{d t}+\left(C E_{e}\right)_{\kappa_{i}}=0,
$$

where $\left(H_{f}\right)_{i}$ denotes the average of $\mathbf{H} \cdot \mathbf{n}_{i}$ where $\mathbf{n}_{i}$ is the unit normal to the face $\kappa_{i}$ and $\left(C E_{e}\right)_{\kappa_{i}}$ is the discrete circulation around the face $\kappa_{i}$.

Let $E$ and $H$ denote vectors of components in $R^{M_{1}}$ and $R^{F_{1}}$, respectively. Then (4.1) and (4.2) (which are exact) suggest the approximations (where it is implied 
that components of $E$ associated with boundary edges are zero, i.e. $\left.E\right|_{\Gamma}=0$ )

$$
\begin{aligned}
\epsilon S^{\prime} \frac{d E}{d t}-C^{\prime} H & =\tilde{J}, \\
\mu S \frac{d H}{d t}+C E & =0,
\end{aligned}
$$

where $\tilde{J} \in R^{M_{1}}$ with components given by the right hand of (4.1). Since $\mu S$ and $\epsilon S^{\prime}$ are invertible, (4.3)-(4.4) is a system of linear ordinary differential equations, and the existence and uniqueness of a solution follow from well-known results.

From (4.4) and (3.7) in Lemma 2 we obtain

$$
\mu \frac{d}{d t}(\mathcal{D} H)=\mu \frac{d}{d t}\left(B_{1}^{T} S H\right)=-B_{1}^{T} C E=0 .
$$

This shows the sense in which $\operatorname{div} H=0$ is satisfied at the discrete level in the covolume scheme.

By subtracting (4.3) from (4.1) we have

$$
\epsilon S^{\prime} \frac{d}{d t}\left(E-E_{f}^{\prime}\right)-C^{\prime}\left(H-H_{e}^{\prime}\right)=0 .
$$

Similarly, from (4.2) and (4.4)

$$
\mu S \frac{d}{d t}\left(H-H_{f}\right)+C\left(E-E_{e}\right)=0
$$

where $(\mathbf{E}, \mathbf{H})$ denotes the exact solution of (2.1)-(2.5) and where, by the boundary condition (2.4),

$$
\left.\eta_{\mathbf{E}}\right|_{\Gamma}=0 \text {. }
$$

Note that the error in the magnetic field $\mathbf{H}$ satisfies the discrete solenoidal condition

$$
\mathcal{D} \dot{\epsilon}_{\mathbf{H}}=0 \text {. }
$$

Multiplying (4.5) by $D \eta_{\mathbf{E}}$ and (4.6) by $D^{\prime} \eta_{\mathbf{H}}$, and adding we obtain

$$
\epsilon\left(\dot{\epsilon}_{\mathbf{E}}^{\prime}, \eta_{\mathbf{E}}\right)_{W^{\prime}}+\mu\left(\dot{\epsilon}_{\mathbf{H}}, \eta_{\mathbf{H}}^{\prime}\right)_{W}=\left(C^{\prime} \eta_{\mathbf{H}}^{\prime}, D \eta_{\mathbf{E}}\right)-\left(C \eta_{\mathbf{E}}, D^{\prime} \eta_{\mathbf{H}}^{\prime}\right),
$$

where the dots denote time differentiations.

The main result in this section is the following theorem.

Theorem 1. Denote by $(E, H)$ the solution of (4.3) and (4.4) and by

$$
(\mathbf{E}, \mathbf{H}) \in W^{1,1}\left(0, T ;\left(W^{1, p}(\Omega)\right)^{3}\right)^{2}
$$

the solution of (2.1)-(2.5) with $p>2$. Then we have the estimate

$$
\begin{array}{r}
\max _{0 \leq t \leq T}\left(\left\|\left(E-E_{e}\right)(t)\right\|_{W^{\prime}}+\left\|\left(H-H_{e}\right)(t)\right\|_{W}\right) \leq K h\left(\|\dot{\mathbf{E}}\|_{L^{1}\left(0, T ;\left(W^{1, p}(\Omega)\right)^{3}\right)}\right. \\
\left.+\|\dot{\mathbf{H}}\|_{L^{1}\left(0, T ;\left(W^{1, p}(\Omega)\right)^{3}\right)}\right) .
\end{array}
$$

To prove this theorem we use (4.9) to get

$$
\begin{aligned}
\epsilon\left(\dot{\eta}_{\mathbf{E}}, \eta_{\mathbf{E}}\right)_{W^{\prime}}+\mu\left(\dot{\eta}_{\mathbf{H}}^{\prime}, \eta_{\mathbf{H}}^{\prime}\right)_{W}= & \epsilon\left(\left(\eta_{\mathbf{E}}-\epsilon_{\mathbf{E}}^{\prime}\right)^{\bullet}, \eta_{\mathbf{E}}\right)_{W}+\mu\left(\left(\eta_{\mathbf{H}}^{\prime}-\epsilon_{\mathbf{H}}\right)^{\bullet}, \eta_{\mathbf{H}}^{\prime}\right)_{W} \\
& +\left(C^{\prime} \eta_{\mathbf{H}}^{\prime}, D \eta_{\mathbf{E}}\right)-\left(C \eta_{\mathbf{E}}, D^{\prime} \eta_{\mathbf{H}}^{\prime}\right) .
\end{aligned}
$$

Since $\mathbf{E} \times\left.\mathbf{n}\right|_{\Gamma}=0$, the components of $\eta_{\mathbf{E}}$ restricted to the boundary are 0 . So Lemma 1 is applied to yield

$$
\left(C^{\prime} \eta_{\mathbf{H}}^{\prime}, D \eta_{\mathbf{E}}\right)-\left(C \eta_{\mathbf{E}}, D^{\prime} \eta_{\mathbf{H}}^{\prime}\right)=0
$$


By

$$
\begin{aligned}
\eta_{\mathbf{E}}-\epsilon_{\mathbf{E}}^{\prime} & =E_{f}-E_{e} \\
\eta_{\mathbf{H}}^{\prime}-\epsilon_{\mathbf{H}} & =H_{f}-H_{e}
\end{aligned}
$$

we obtain from (4.9)

$$
\frac{1}{2} \frac{d}{d t}\left(\left\|(\mu)^{\frac{1}{2}} \eta_{\mathbf{H}}^{\prime}\right\|_{W}^{2}+\left\|(\epsilon)^{\frac{1}{2}} \eta_{\mathbf{E}}\right\|_{W^{\prime}}^{2}\right)=\mu\left(\left(H_{f}-H_{e}\right)^{\bullet}, \eta_{\mathbf{H}}^{\prime}\right)_{W}+\epsilon\left(\left(E_{f}-E_{e}\right)^{\bullet}, \eta_{\mathbf{E}}\right)_{W^{\prime}},
$$

where ()$^{\bullet}$ denotes time differentiations. Integrating (4.10) from 0 to $T$ and using Cauchy's inequality we obtain

$$
\begin{aligned}
& \left(\left\|(\mu)^{\frac{1}{2}} \eta_{\mathbf{H}}^{\prime}(T)\right\|_{W}^{2}+\left\|(\epsilon)^{\frac{1}{2}} \eta_{\mathbf{E}}(T)\right\|_{W^{\prime}}^{2}\right) \\
& \leq 2 \int_{0}^{T}\left(\left\|(\mu)^{\frac{1}{2}}\left(H_{e}-H_{f}\right)^{\bullet}\right\|_{W}\left\|(\mu)^{\frac{1}{2}} \eta_{\mathbf{H}}^{\prime}(s)\right\|_{W}\right. \\
& \left.\quad+\left\|(\epsilon)^{\frac{1}{2}}\left(E_{e}-E_{f}\right)^{\bullet}\right\|_{W^{\prime}}\left\|(\epsilon)^{\frac{1}{2}} \eta_{\mathbf{E}}(s)\right\|_{W^{\prime}}\right) d s .
\end{aligned}
$$

Let $t^{*}$ be such that

$$
\left(\left\|(\mu)^{\frac{1}{2}} \eta_{\mathbf{H}}^{\prime}\left(t^{*}\right)\right\|_{W}+\left\|(\epsilon)^{\frac{1}{2}} \eta_{\mathbf{E}}\left(t^{*}\right)\right\|_{W^{\prime}}\right)=\max _{0 \leq t \leq T}\left(\left\|(\mu)^{\frac{1}{2}} \eta_{\mathbf{H}}(t)\right\|_{W}+\left\|(\epsilon)^{\frac{1}{2}} \eta_{\mathbf{E}}(t)\right\|_{W^{\prime}}\right) .
$$

Then

$$
\begin{gathered}
\left(\left\|\mu^{\frac{1}{2}} \eta_{\mathbf{H}}^{\prime}\left(t^{*}\right)\right\|_{W}+\left\|\epsilon^{\frac{1}{2}} \eta_{\mathbf{E}}\left(t^{*}\right)\right\|_{W^{\prime}}\right)^{2} \leq 2\left(\left\|\mu^{\frac{1}{2}} \eta_{\mathbf{H}}^{\prime}(T)\right\|_{W}^{2}+\left\|\epsilon^{\frac{1}{2}} \eta_{\mathbf{E}}(T)\right\|_{W^{\prime}}^{2}\right) \\
\leq 4 \int_{0}^{t^{*}}\left(\left\|\mu^{\frac{1}{2}}\left(H_{e}-H_{f}\right)^{\bullet}\right\|_{W}\left\|\mu^{\frac{1}{2}} \eta_{\mathbf{H}}^{\prime}(s)\right\|_{W}\right. \\
\left.\quad+\left\|\epsilon^{\frac{1}{2}}\left(E_{e}-E_{f}\right)^{\bullet}\right\|_{W^{\prime}}\left\|\epsilon^{\frac{1}{2}} \eta_{\mathbf{E}}(s)\right\|_{W^{\prime}}\right) d s \\
\leq 4 \int_{0}^{T}\left(\left\|\mu^{\frac{1}{2}}\left(H_{e}-H_{f}\right)^{\bullet}\right\|_{W}\left\|\mu^{\frac{1}{2}} \eta_{\mathbf{H}}^{\prime}(s)\right\|_{W}\right. \\
\left.+\left\|\epsilon^{\frac{1}{2}}\left(E_{e}-E_{f}\right)^{\bullet}\right\|_{W^{\prime}}\left\|(\epsilon)^{\frac{1}{2}} \eta_{\mathbf{E}}(s)\right\|_{W^{\prime}}\right) d s .
\end{gathered}
$$

So it follows that

$$
\begin{aligned}
\max _{0 \leq t \leq T}\left(\left\|(\mu)^{\frac{1}{2}} \eta_{\mathbf{H}}^{\prime}(t)\right\|_{W}+\left\|(\epsilon)^{\frac{1}{2}} \eta_{\mathbf{E}}(t)\right\|_{W^{\prime}}\right) \\
\quad \leq 4 \int_{0}^{T}\left(\left\|(\mu)^{\frac{1}{2}}\left(H_{e}-H_{f}\right)^{\bullet}\right\|_{W}+\left\|(\epsilon)^{\frac{1}{2}}\left(E_{e}-E_{f}\right)^{\bullet}\right\|_{W^{\prime}}\right) d s .
\end{aligned}
$$

Theorem 1 will follow from Lemma 3 :

Lemma 3. Assume that $\dot{\mathbf{E}}, \dot{\mathbf{H}} \in\left(W^{1, p}(\Omega)\right)^{3}, p>2$. Then there exists a generic constant $K$, such that

$$
\begin{aligned}
& \left\|\left(H_{e}-H_{f}\right)^{\bullet}\right\|_{W} \leq K h|\dot{\mathbf{H}}|_{\left(W^{1, p}(\Omega)\right)^{3}}, \\
& \left\|\left(E_{e}-E_{f}\right)^{\bullet}\right\|_{W^{\prime}} \leq K h|\dot{\mathbf{E}}|_{\left(W^{1, p}(\Omega)\right)^{3}},
\end{aligned}
$$

where

$$
K \leq K^{\prime} \max \left(\max _{i}\left(\frac{s_{i}}{{h^{\prime}}_{i}^{2}}\right), \max _{i}\left(\frac{s_{i}^{\prime}}{h_{i}^{2}}\right)\right)
$$

and $K^{\prime}$ is a constant. 
Proof. For the primal face $\kappa_{i}$,

$$
\left(H_{e}-H_{f}\right)^{\bullet}=\frac{1}{h_{i}^{\prime}} \int_{\sigma_{i}^{\prime}} \dot{\mathbf{H}} \cdot \mathbf{n} d l-\frac{1}{s_{i}} \int_{\kappa_{i}} \dot{\mathbf{H}} \cdot \mathbf{n} d x .
$$

By the Sobolev embedding theorem, $V: \mathbf{H} \rightarrow\left(\dot{H}_{e}-\dot{H}_{f}\right)$ defines a bounded linear functional on $\left(W^{1, p}\left(\kappa_{j}\right)\right)^{3}$. For constant $\dot{\mathbf{H}}$ this linear functional vanishes in the union of two tetrahedra that share the same face $\kappa_{i}$ and consequently

$$
\left|\left(H_{e}-H_{f}\right)^{\bullet}\right| \leq K\left(\tau_{i} \cup \tau_{l}\right)|\dot{\mathbf{H}}|_{W^{1, p}\left(\tau_{i} \cup \tau_{l}\right)^{3}},
$$

where $K\left(\tau_{i} \cup \tau_{l}\right)$ is a constant. In order to estimate $K\left(\tau_{i} \cup \tau_{l}\right)$, we use a standard scale change argument as follows.

Let the primal face $\kappa_{i}=\tau_{i} \cap \tau_{l}$ be in the $x y$ plane so that $\sigma_{i}^{\prime}$ is parallel to the $z$ axis, changing the coordinates by

$$
\left(\begin{array}{l}
x \\
y
\end{array}\right)=A\left(\begin{array}{c}
x^{\prime} \\
y^{\prime}
\end{array}\right), \quad z=h_{1} z^{\prime}
$$

where $A$ is a $2 \times 2$ matrix, it results that $K\left(\tau_{i} \cup \tau_{l}\right)$ depends on the quantity

$$
\max \left(\left\|A^{-1}\right\|_{2}^{-1}\left(|\operatorname{det} A| h_{1}\right)^{-\frac{1}{p}}, \frac{h_{1}^{\frac{1}{p^{\prime}}}}{(\operatorname{det} A)^{\frac{1}{p}}}\right)
$$

where $\frac{1}{p}+\frac{1}{p^{\prime}}=1$. Let $h_{i}^{\prime}$ denote the length of the co-edge and $s_{i}$ the area of the primal face. A further calculation then shows that $|\operatorname{det} A|=c_{1} s_{i}, h_{1}=c_{2} h_{i}^{\prime}$ so that

$$
\left\|A^{-1}\right\|_{2}^{-1}\left(|\operatorname{det} A| h_{1}\right)^{-\frac{1}{p}}=c_{3} \frac{\sqrt{\lambda_{\min }}\left(A^{T} A\right)}{\left(s_{i} h_{i}^{\prime}\right)^{\frac{1}{p}}} \leq c_{3} \frac{\sqrt[4]{\operatorname{det}\left(A^{T} A\right)}}{\left(s_{i} h_{i}^{\prime}\right)^{\frac{1}{p}}}=c_{4} \frac{s_{i}^{\frac{1}{2}-\frac{1}{p}}}{h_{i}^{\prime \frac{1}{p}}},
$$

where $c_{i}, i=1, \cdots, 4$, are independent of $h_{i}^{\prime}$ and $s_{i}$, and $\lambda_{\min }$ is the least eigenvalue of the positive definite matrix $A^{T} A$.

Collecting these results, $K\left(\tau_{i} \cup \tau_{l}\right)$ is bounded by

$$
K\left(\tau_{i} \cup \tau_{l}\right) \leq \max _{i}\left(\frac{s_{i}^{\frac{1}{2}-\frac{1}{p}}}{h_{i}^{\prime \frac{1}{p}}}, \frac{h_{i}^{\prime \frac{1}{p^{\prime}}}}{s_{i}^{\frac{1}{p}}}\right) .
$$

Now we have

$$
\begin{aligned}
\left\|\left(H_{e}-H_{f}\right)^{\bullet}\right\|_{W}^{2} & =\sum_{i=1}^{F} s_{i} h_{i}^{\prime}\left|\left(H_{e}-H_{f}\right)^{\bullet}\right|^{2} \\
& \leq K_{1} \sum_{i=1}^{F} s_{i} h_{i}^{\prime} \max _{i}\left(\frac{s_{i}^{1-\frac{2}{p}}}{h_{i}^{\prime \frac{2}{p}}}, \frac{h_{i}^{\prime \frac{2}{p^{\prime}}}}{s_{i}^{\frac{2}{p}}}\right)|\dot{\mathbf{H}}|_{\left(W^{1, p}\left(\tau_{i} \cup \tau_{l}\right)\right)^{3}}^{2} \\
& \leq K_{2} \sum_{i=1}^{F} h^{5-\frac{6}{p}}|\dot{\mathbf{H}}|_{\left(W^{1, p}\left(\tau_{i} \cup \tau_{l}\right)\right)^{3}}^{2},
\end{aligned}
$$


where $K_{2}$ depends only on $\max \left(\frac{s_{i}}{{h^{\prime}}^{\prime}}\right)$. By Holder's inequality and since $h^{3} F \leq$ $K^{\prime}$ measure $(\Omega)$ we obtain

$$
\begin{aligned}
\left\|\left(H_{e}-H_{f}\right)^{\bullet}\right\|_{W}^{2} & \leq K_{2} h^{5-\frac{6}{p}}\left(\sum_{i=1}^{F}|\dot{\mathbf{H}}|_{\left(W^{1, p}\left(\tau_{i} \cup \tau_{l}\right)\right)^{3}}^{p}\right)^{\frac{2}{p}}\left(\sum_{i=1}^{F} 1\right)^{\frac{p-2}{p}} \\
& =K_{3} h^{5-\frac{6}{p}} F^{\frac{p-2}{p}}|\dot{\mathbf{H}}|_{\left(W^{1, p}(\Omega)\right)^{3}}^{2} \\
& \leq K h^{2}|\dot{\mathbf{H}}|_{\left(W^{1, p}(\Omega)\right)^{3} .}^{2}
\end{aligned}
$$

The proof of the estimate for $\left\|\left(E_{e}-E_{f}\right)^{\bullet}\right\|_{W^{\prime}}$ is similar.

From the estimate above we know the constant $K$ depends on the quantity

$$
\max \left(\max _{i}\left(\frac{s_{i}}{h_{i}^{\prime 2}}\right), \max _{i}\left(\frac{s_{i}^{\prime}}{h_{i}^{2}}\right)\right) .
$$

Theorem 1 now follows from (4.11) and Lemma 3.

\section{The FUlly Discrete Problem}

There are many possible time stepping methods that can be applied to (4.3) and (4.4). We will discuss a leapfrog scheme which is very popular in computational electromagnetics (see [18]). In this scheme we approximate $\mathbf{E}(t)$ at times $t_{n}=$ $n \triangle t, 0 \leq n<\infty$ with a vector $\left\{\mathcal{E}^{n}\right\}_{n=0}^{\infty}$, and $\mathbf{H}(t)$ at time $t_{n+\frac{1}{2}}$ with a vector $\left\{\mathcal{H}^{n+\frac{1}{2}}\right\}_{n=0}^{\infty}$. The initial value $\mathcal{H}^{\frac{1}{2}}$ can be computed using, for example, a Taylor expansion and the given equations $(2.1)-(2.2)$. Given $\left(\mathcal{E}^{n}, \mathcal{H}^{n+\frac{1}{2}}\right)_{n \geq 0}$, the next approximation $\left(\mathcal{E}^{n+1}, \mathcal{H}^{n+\frac{3}{2}}\right)$ is obtained by solving the equations

$$
\begin{gathered}
\epsilon S^{\prime}\left(\mathcal{E}^{n+1}-\mathcal{E}^{n}\right)-\triangle t C^{\prime} \mathcal{H}^{n+\frac{1}{2}}=\tilde{J}^{n+\frac{1}{2}} \\
\mu S\left(\mathcal{H}^{n+\frac{3}{2}}-\mathcal{H}^{n+\frac{1}{2}}\right)+\triangle t C \mathcal{E}^{n+1}=0
\end{gathered}
$$

where

$$
\tilde{J}^{n+\frac{1}{2}}=\int_{n \triangle t}^{(n+1) \triangle t} \tilde{J} d t .
$$

(5.1), (5.2) is an explicit scheme, so the existence and uniqueness of a solution are apparent. Using the error functions defined in the last section, we can rewrite $(5.1)-(5.2)$ as

$$
\begin{gathered}
\epsilon S^{\prime}\left(\epsilon_{\mathbf{E}}^{n+1}-\epsilon_{\mathbf{E}}^{n}\right)=(\triangle t) C^{\prime} \eta_{\mathbf{H}}^{n+\frac{1}{2}}+G^{n}, \\
\mu S\left(\epsilon_{\mathbf{H}}^{n+\frac{3}{2}}-\epsilon_{\mathbf{H}}^{n+\frac{1}{2}}\right)=-(\triangle t) C \eta_{\mathbf{E}}^{n+1}+\bar{G}_{n} .
\end{gathered}
$$

By a direct computation, $G^{n}$ and $\bar{G}^{n}$ are given by

$$
\begin{gathered}
G^{n}=\tilde{J}^{n+\frac{1}{2}}-\epsilon S^{\prime}\left(E_{f}^{n+1}-E_{f}^{n}\right)+\triangle t C^{\prime} H_{e}^{n+\frac{1}{2}}, \\
\bar{G}^{n}=\mu S\left(H_{f}^{n+\frac{3}{2}}-H_{f}^{n+\frac{1}{2}}\right)-\triangle t C E_{e}^{n+1} .
\end{gathered}
$$

Our main estimate for the fully discrete scheme is then given in the following theorem: 
Theorem 2. Let $\left(\mathcal{E}^{n}, \mathcal{H}^{n+\frac{1}{2}}\right)_{n \geq 0}^{N-1}$ denote the solution of (5.1)-(5.2), and let

$$
(\mathbf{E}, \mathbf{H}) \in\left(H^{1}\left(0, T ;\left(W^{1, p}(\Omega)\right)^{3}\right)\right)^{2}
$$

denote the solution of $(2.1)-(2.4), p>2$. Under the stability condition

$$
c \Delta t<\frac{\min \left(h_{i j}\right)}{\sqrt{M_{3}} M_{2}{ }^{\frac{3}{2}}},
$$

where $c=(\epsilon \mu)^{-\frac{1}{2}}$ is the speed of the light in the medium, $M_{2}$ is the maximum of the ratios of the maximum to minimum side-lengths over the union of adjacent tetrahedra, and $M_{3}$ is the maximum number of edges over all co-faces, we have the following error estimate for the fully discrete scheme (5.1)-(5.2)

$$
\begin{aligned}
& \max _{0 \leq i \leq N-1}\left(\epsilon\left\|\mathcal{E}^{i}-E_{e}^{i}\right\|_{W^{\prime}}+\mu\left\|\mathcal{H}^{i+\frac{1}{2}}-H_{e}^{i+\frac{1}{2}}\right\|_{W}\right) \\
& \quad \leq K h\left(\|\mathbf{E}\|_{H^{1}\left(0, T ;\left(W^{1, p}(\Omega)\right)^{3}\right)}+\|\mathbf{H}\|_{H^{1}\left(0, T ;\left(W^{1, p}(\Omega)\right)^{3}\right)}\right) .
\end{aligned}
$$

Proof. Multiplying (5.3) by $D\left(\eta_{\mathbf{E}}^{n}+\eta_{\mathbf{E}}^{n+1}\right)$ and (5.4) by $D^{\prime}\left(\eta_{\mathbf{H}}^{n+\frac{3}{2}}+\eta_{\mathbf{H}}^{n+\frac{1}{2}}\right)$, respectively, and adding all these equations from $n=0,1, \cdots, N-1$ gives

$$
\begin{aligned}
\epsilon\left\|\eta_{\mathbf{E}}^{N}\right\|_{W^{\prime}}^{2}+\mu\left\|\eta_{\mathbf{H}}^{N-\frac{1}{2}}\right\|_{W}^{2}= & \Delta t\left(C^{\prime} \eta_{\mathbf{H}}^{N-\frac{1}{2}}, D \eta_{\mathbf{E}}^{N}\right) \\
& +\sum_{i=0}^{N-1} \epsilon\left(\delta_{\mathbf{E}}^{i+1}-\delta_{\mathbf{E}}^{i}, \eta_{\mathbf{E}}^{i}\right)_{W^{\prime}}+\sum_{i=0}^{N-2} \mu\left(\delta_{\mathbf{H}}^{i+\frac{3}{2}}-\delta_{\mathbf{H}}^{i+\frac{1}{2}}, \eta_{\mathbf{H}}^{i+\frac{1}{2}}\right)_{W} \\
& +\sum_{i=0}^{N-1}\left(\left(G^{i}, D\left(\eta_{\mathbf{E}}^{i}+\eta_{\mathbf{E}}^{i+1}\right)\right)+\sum_{i=0}^{N-2}\left(\bar{G}^{i}, D^{\prime}\left(\eta_{\mathbf{H}}^{i+\frac{3}{2}}+\eta_{\mathbf{H}}^{i+\frac{1}{2}}\right)\right)\right) .
\end{aligned}
$$

The proof has three steps:

(i) First,

$$
\begin{aligned}
\triangle t\left(C^{\prime} \eta_{\mathbf{H}}^{N-\frac{1}{2}}, D \eta_{\mathbf{E}}^{N}\right) & =\triangle t\left(C^{\prime}\left(S D^{\prime}\right)^{-\frac{1}{2}}\left(\left(S D^{\prime}\right)^{\frac{1}{2}} \eta_{\mathbf{H}}^{N-\frac{1}{2}}\right),\left(D S^{\prime-1}\right)^{\frac{1}{2}}\left(\left(D S^{\prime}\right)^{\frac{1}{2}} \eta_{\mathbf{E}}^{N}\right)\right) \\
& \leq \triangle t\left\|\left(S D^{\prime}\right)^{-\frac{1}{2}} C^{\prime}\left(D S^{\prime-1}\right)^{\frac{1}{2}}\right\|_{2}\left\|\eta_{\mathbf{H}}^{N-\frac{1}{2}}\right\|_{W}\left\|\eta_{\mathbf{E}}^{N}\right\|_{W^{\prime}}
\end{aligned}
$$

From algebra, $\left\|\left(S D^{\prime}\right)^{-\frac{1}{2}} C^{\prime}\left(D S^{\prime-1}\right)^{\frac{1}{2}}\right\|_{2}$ is the largest singular value of the matrix and by Gershgorin's theorem

$$
\left\|\left(S D^{\prime}\right)^{-\frac{1}{2}} C^{\prime}\left(D S^{\prime-1}\right)^{\frac{1}{2}}\right\|_{2} \leq \max \left(\frac{\max _{i j}\left(h_{i j}\right)^{\frac{3}{2}}}{\min _{i j}\left(h_{i j}\right)^{\frac{5}{2}}}\right)\left(2 \sqrt{M_{3}}\right),
$$

where maximum above is taken over all of the union of adjacent tetrahedra. Combining the results gives

$$
\begin{aligned}
\triangle t\left(C^{\prime} \eta_{\mathbf{H}}^{N-\frac{1}{2}}, D \eta_{\mathbf{E}}^{N}\right) & \leq \triangle t \frac{2 \sqrt{M_{3}} M_{2}^{\frac{3}{2}}}{\min \left(h_{i j}\right)}\left\|\eta_{\mathbf{H}}^{N-\frac{1}{2}}\right\|_{W}\left\|\eta_{\mathbf{E}}^{N}\right\|_{W^{\prime}} \\
& \leq \triangle t \frac{\sqrt{M_{3}} M_{2}^{\frac{3}{2}}}{\min \left(h_{i j}\right)} c\left(\epsilon\left\|\eta_{\mathbf{E}}^{N}\right\|_{W^{\prime}}^{2}+\mu\left\|\eta_{\mathbf{H}}^{N-\frac{1}{2}}\right\|_{W}^{2}\right) \\
& <\left(\epsilon\left\|\eta_{\mathbf{E}}^{N}\right\|_{W^{\prime}}^{2}+\mu\left\|\eta_{\mathbf{H}}^{N-\frac{1}{2}}\right\|_{W}^{2}\right) .
\end{aligned}
$$


(ii) $\delta_{\mathbf{E}}^{i+1}-\delta_{\mathbf{E}}^{i}$ can be estimated in the following way. We have, by integrating in time

$$
\left|\delta_{\mathbf{E}}^{i+1}-\delta_{\mathbf{E}}^{i}\right| \leq\left\|\dot{\delta}_{\mathbf{E}}\right\|_{L^{1}(i \triangle t \leq t \leq(i+1) \triangle t)}
$$

So

$$
\begin{aligned}
\left\|\delta_{\mathbf{E}}^{i+1}-\delta_{\mathbf{E}}^{i}\right\|_{W^{\prime}}^{2} & =\sum_{k=1}^{M_{1}} s_{k}^{\prime} h_{k}\left|\left(\delta_{\mathbf{E}}^{i+1}\right)_{k}-\left(\delta_{\mathbf{E}}^{i}\right)_{k}\right|^{2} \\
& \leq \sum_{k=1}^{M_{1}} s_{k}^{\prime} h_{k}\left(\int_{i \triangle t}^{(i+1) \triangle t}\left|\left(\dot{\delta}_{\mathbf{E}}\right)_{k}\right| d s\right)^{2} \\
& \leq \triangle t \int_{i \triangle t}^{(i+1) \triangle t} \sum_{k=1}^{M_{1}} s_{k}^{\prime} h_{k}\left|\left(\dot{\dot{\delta}_{\mathbf{E}}}\right)_{k}\right|^{2} d s \\
& =\triangle t \int_{i \triangle t}^{(i+1) \triangle t}\left\|\dot{\delta}_{\mathbf{E}}\right\|_{W^{\prime}}^{2} d s .
\end{aligned}
$$

By Lemma 3

$$
\left\|\delta_{\mathbf{E}}^{i+1}-\delta_{\mathbf{E}}^{i}\right\|_{W^{\prime}} \leq K_{1} h \sqrt{\triangle t}|| \dot{\mathbf{E}} \|_{L^{2}\left((i \triangle t,(i+1) \triangle t) ;\left(W^{1, p}(\Omega)\right)^{3}\right)} .
$$

Similarly,

$$
\left\|\delta_{\mathbf{H}}^{i+\frac{3}{2}}-\delta_{\mathbf{H}}^{i+\frac{1}{2}}\right\|_{W} \leq K_{1} h \sqrt{\triangle t}|| \dot{\mathbf{H}} \|_{L^{2}\left((i \triangle t,(i+1) \triangle t) ;\left(W^{1, p}(\Omega)\right)^{3}\right)} .
$$

(iii) We have from the definition of $\|\cdot\|_{W^{\prime}}$

$$
\left(G^{i}, D\left(\eta_{\mathbf{E}}^{i}+\eta_{\mathbf{E}}^{i+1}\right)\right) \leq 2\left\|\left(S^{\prime-1}\right) G^{i}\right\|_{W^{\prime}}\left(\left\|\eta_{\mathbf{E}}^{i}\right\|_{W^{\prime}}+\left\|\eta_{\mathbf{E}}^{i+1}\right\|_{W^{\prime}}\right) .
$$

From (5.5)

$$
G_{l}^{i}=-\int_{i \triangle t}^{(i+1) \triangle t}\left(\left(C^{\prime} H_{e}\right)_{l}+\triangle t\left(C^{\prime} H_{e}^{i+\frac{1}{2}}\right)_{l}\right) d s,
$$

where the subscript corresponds the $l$ th dual edge. By the quadrature rule, $G_{l}^{i}$ vanishes for constant $\left(C^{\prime} H_{e}\right)_{l}$ in time, so

$$
\left|G_{l}^{i}\right| \leq K_{2}\left\|\left(C^{\prime} \dot{\mathbf{H}}_{e}\right)\right\|_{L^{1}(i \triangle t \leq t \leq(i+1) \triangle t)},
$$

where, by a scale change, $K_{2}$ varies as $\triangle t$. By the Bramble-Hilbert lemma

$$
\left|C^{\prime} \dot{\mathbf{H}}_{e}\right| \leq K_{3}|\dot{\mathbf{H}}|_{\left(W^{1, p}\left(\tau_{l}\right)\right)^{3}},
$$

where, by scaling, $K_{3}$ varies as $h^{2-\frac{3}{p}}$. So

$$
\left|G_{l}^{i}\right| \leq K_{4} h^{2-\frac{3}{p}} \triangle t \int_{i \triangle t}^{(i+1) \triangle t}|\dot{\mathbf{H}}|_{\left(W^{1, p}\left(\tau_{l}\right)\right)^{3}} d s .
$$

Using Cauchy's inequality

$$
\left|G_{l}^{i}\right|^{2} \leq K_{4} h^{4-\frac{6}{p}}(\triangle t)^{3} \int_{i \triangle t}^{(i+1) \triangle t}|\dot{\mathbf{H}}|_{\left(W^{1, p}\left(\tau_{l}\right)\right)^{3}}^{2} d s
$$


and

$$
\begin{aligned}
\sum_{l=1}^{F_{1}}\left|G_{l}^{i}\right|^{2} & \leq K_{4} h^{4-\frac{6}{p}}(\triangle t)^{3} \int_{i \triangle t}^{(i+1) \triangle t} \sum_{l=1}^{F_{1}}|\dot{\mathbf{H}}|_{\left(W^{1, p}\left(\tau_{l}\right)\right)^{3}}^{2} d s \\
& \leq K_{4} h^{4-\frac{6}{p}}(\triangle t)^{3}\left(\sum_{l=1}^{F_{1}} 1\right)^{1-\frac{2}{p}} \int_{i \triangle t}^{(i+1) \triangle t}\left(\sum_{l=1}^{F_{1}}|\dot{\mathbf{H}}|_{\left(W^{1, p}\left(\tau_{l}\right)\right)^{3}}^{p}\right)^{\frac{2}{p}} d s
\end{aligned}
$$

from Holder's inequality. Using $(5.7)$ to estimate $\triangle t$ we obtain

$$
\left(G^{i}, G^{i}\right) \leq K_{4} h^{3} \triangle t|\dot{\mathbf{H}}|_{L^{2}\left((i \triangle t,(i+1) \triangle t) ;\left(W^{1, p}(\Omega)\right)^{3}\right)}^{2}
$$

and from the definition of $\|\cdot\|_{W^{\prime}}$

$$
\left\|\left(S^{\prime-1}\right) G^{i}\right\|_{W^{\prime}} \leq K_{5} h \sqrt{\triangle t} \mid \mathbf{H} \|_{H^{1}\left((i \triangle t,(i+1) \triangle t) ;\left(W^{1, p}(\Omega)\right)^{3}\right)} .
$$

By Cauchy's inequality $\sum_{i=1}^{N} a_{i} \leq \sqrt{N}\left(\sum_{i=1}^{N} a_{i}^{2}\right)^{\frac{1}{2}}$ and $N \triangle t=T$

$$
\sum_{i=1}^{N}\left\|\left(S^{\prime-1}\right) G^{i}\right\|_{W^{\prime}} \leq K_{7} h\|\mathbf{H}\|_{H^{1}\left(0, T ;\left(W^{1, p}(\Omega)\right)^{3}\right)}
$$

Similarly,

$$
\left\|S^{-1} \bar{G}^{i}\right\|_{W} \leq K_{8} h \sqrt{\triangle t}\|\mathbf{E}\|_{H^{1}\left((i \triangle t,(i+1) \triangle t) ;\left(W^{1, p}(\Omega)\right)^{3}\right)}
$$

and

$$
\sum_{i=1}^{N}\left\|\left(S^{-1}\right) \bar{G}^{i}\right\|_{W} \leq K_{9} h\|\mathbf{E}\|_{H^{1}\left(0, T ;\left(W^{1, p}(\Omega)\right)^{3}\right)} .
$$

Collecting the terms from (i)-(iii), we obtain finally

$$
\begin{aligned}
& \max _{0 \leq i \leq N-1}\left(\epsilon\left\|\eta_{\mathbf{E}}^{i}\right\|_{W^{\prime}}+\mu\left\|\eta_{\mathbf{H}}^{i+\frac{1}{2}}\right\|_{W}\right) \\
& \quad \leq K h\left(\|\mathbf{E}\|_{H^{1}\left(0, T ;\left(W^{1, p}(\Omega)\right)^{3}\right)}+\|\mathbf{H}\|_{H^{1}\left(0, T ;\left(W^{1, p}(\Omega)\right)^{3}\right)}\right)
\end{aligned}
$$

and this proves (5.8).

\section{On RECTANGUlar MEShes}

The covolume scheme can be extended to rectangular meshes. In this case both primal and co-face are rectangles and orthogonal to each other. All the duality relations discussed in Section 2 are preserved. This method is the standard Yee scheme [18].

Motivated by [11], we will show in this section that on nonuniform but rectangular grids (a.k.a. graded grids) with maximum size $h$ the covolume approximation of tangential components of electric field $\mathbf{E}$ and normal components of magnetic field $\mathbf{H}$ are second order in space in $\|\cdot\|_{W^{\prime}}$ and $\|\cdot\|_{W}$, respectively. Here we only need $(\mathbf{E}, \mathbf{H})$ in $\left(L^{1}\left(0, T ;\left(H^{3}(\Omega)\right)^{3}\right)\right)^{2}$. This improves the norm used in [11], where $(\mathbf{E}, \mathbf{H})$ in $\left(L^{1}\left(0, T ;\left(C^{3}(\Omega)\right)^{3}\right)\right)^{2}$ was assumed.

Using Lemma 1 and the error functions introduced in Section 4, we can rewrite (4.9) as

$$
\frac{1}{2} \frac{d}{d t}\left(\left\|\epsilon_{\mathbf{H}}\right\|_{W}^{2}+\left\|\eta_{\mathbf{E}}\right\|_{W^{\prime}}^{2}\right)=\left(\dot{\delta}_{\mathbf{E}}, \eta_{\mathbf{E}}\right)_{W^{\prime}}-\left(\dot{\epsilon}_{\mathbf{H}}, \delta_{\mathbf{H}}\right)_{W}
$$

We need here two technical lemmas to estimate the terms on the right side of (6.1). 
Lemma 4. There exists $u(t) \in R^{F_{1}}$ such that each component of $u$ is a continuous linear functional of the magnetic field $\mathbf{H}$ and

$$
\left(\dot{\epsilon}_{\mathbf{H}}, \delta_{\mathbf{H}}\right)_{W}=\left(\dot{\epsilon}_{\mathbf{H}}, u\right)_{W}
$$

with

$$
\max \left(\|u\|_{W},\|\dot{u}\|_{W}\right) \leq K h^{2}\|\mathbf{H}\|_{\left(H^{3}(\Omega)\right)^{3}} .
$$

Lemma 5. There exists $v^{1}(t) \in R^{M_{1}}, v^{2}(t) \in R^{F_{1}}$ such that each component of $v^{1}$ and $v^{2}$ is a continuous linear functional of the electric field $\mathbf{E}$ and

$$
\left(\dot{\delta}_{\mathbf{E}}, \eta_{\mathbf{E}}\right)_{W^{\prime}}=\left(\dot{v}^{1}, \eta_{\mathbf{E}}\right)_{W^{\prime}}+\left(\dot{\epsilon}_{\mathbf{H}}, \dot{v}^{2}\right)_{W}
$$

with

$$
\left\|\dot{v}^{1}\right\|_{W^{\prime}} \leq K h^{2}|\mathbf{E}|_{\left(H^{3}(\Omega)\right)^{3}}, \quad \max \left(\left\|\dot{v}^{2}\right\|_{W},\left\|\ddot{v}^{2}\right\|_{W}\right) \leq K h^{2}\|\mathbf{H}\|_{\left(H^{3}(\Omega)\right)^{3}} .
$$

Assuming these two lemmas we can prove

Theorem 3. Suppose that $(\mathbf{E}, H) \in L^{1}\left(0, T ;\left(H^{3}(\Omega)\right)^{3}\right)^{2}$ satisfies (2.1)-(2.5), and denote by $(E, H)$ the solution of (4.3)-(4.4) on nonuniform grids with maximum grid size $h$. Then

$$
\max _{0 \leq t \leq T}\left(\left\|\left(E-E_{e}\right)(t)\right\|_{W^{\prime}}+\left\|\left(H-H_{f}\right)(t)\right\|_{W}\right) \leq K h^{2}\|(\mathbf{E}, H)\|_{\left(L^{1}\left(0, T ;\left(H^{3}(\Omega)\right)^{3}\right)\right)^{2}} .
$$

Proof of Theorem 3. Substituting (6.2) and (6.4) into (6.1) and integrating from 0 to $t_{1}$,

$$
\begin{aligned}
\frac{1}{2}\left(\left\|\epsilon_{\mathbf{H}}\right\|_{W}^{2}+\left\|\eta_{\mathbf{E}}\right\|_{W^{\prime}}^{2}\right)\left(t_{1}\right)=\left(\epsilon_{\mathbf{H}}, u-\dot{v}^{2}\right)_{W}\left(t_{1}\right) & \\
& +\int_{0}^{t_{1}}\left(\dot{v}^{1}, \eta_{\mathbf{E}}\right)_{W^{\prime}}(\tau) d \tau+\int_{0}^{t_{1}}\left(\epsilon_{\mathbf{H}}, \dot{u}-\ddot{v}^{2}\right)_{W}(\tau) d \tau .
\end{aligned}
$$

Applying Lemma 4, Lemma 5 and Cauchy's inequality proves (6.6).

The method of proving Lemma 4 is to notice that if $h_{1} \neq h_{2}$, the quadrature rule

$$
\int_{-h_{1}}^{h_{2}} f(x) d x \approx\left(h_{1}+h_{2}\right) f(0)
$$

is exact for constant functions, but after adding the correction term

$$
\frac{1}{2}\left(h_{2}^{2} f\left(h_{2}\right)-h_{1}^{2} f^{\prime}\left(-h_{1}\right)\right)
$$

it is exact for linear polynomials. In the proof below we will manipulate these first order correction terms to make them a discrete gradient.

Proof of Lemma 4. For primal face $\kappa_{i},\left(\delta_{\mathbf{H}}\right)_{i}$, the difference between the average of $\mathbf{H} \cdot \mathbf{n}_{i}$ over the face $\kappa_{i}$ and the average of the same quantity along the co-edge $\sigma_{i}^{\prime}=\overline{O O^{\prime}}$ orthogonal to the face $\kappa_{i}$ (see Figure 2), vanishes for the constant field H. So we write $\left(\delta_{\mathbf{H}}\right)_{i}$ as

$$
\overline{O O^{\prime}}\left(\delta_{\mathbf{H}}\right)_{i}=\overline{O O^{\prime}} u_{i}+\tilde{u}_{i}
$$

where the first order correction term $\tilde{u}_{i}$ is

$$
\tilde{u}_{i}:=\frac{1}{2}\left({\overline{O P_{1}}}^{2} \mathbf{H}_{3 z}+h_{x}^{2} \mathbf{H}_{1 x}+h_{y}^{2} \mathbf{H}_{2 y}\right)(O)-\frac{1}{2}\left({\overline{O^{\prime} P_{1}}}^{2} \mathbf{H}_{3 z}+h_{x}^{2} \mathbf{H}_{1 x}+h_{y}^{2} \mathbf{H}_{2 y}\right)\left(O^{\prime}\right)
$$




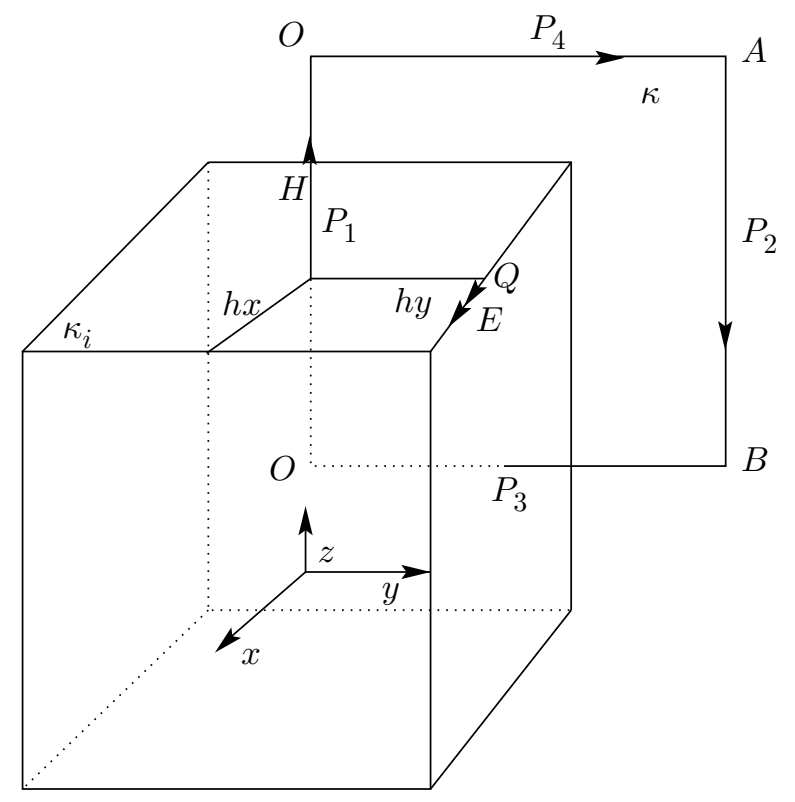

Figure 2

and $u_{i}$ from (6.7) vanishes for piecewise linear polynomial functions. We remark that $\tilde{u}_{i}$ was formed in the way indicated above since there are three co-edges emanating from the center $O$. Note also that $\tilde{u}_{i}$ is a discrete gradient form, i.e., $\tilde{u}=B_{1} \phi$ for some $\phi \in R^{T}$. Using (3.8) in Lemma 2,

$$
\begin{aligned}
\left(\dot{\epsilon}_{\mathbf{H}}, \delta_{\mathbf{H}}\right)_{W} & =\left(\dot{\epsilon}_{\mathbf{H}}, u\right)_{W}+\left(\dot{\epsilon}_{\mathbf{H}}, D^{\prime-1} \tilde{u}\right)_{W} \\
& =\left(\dot{\epsilon}_{\mathbf{H}}, u\right)_{W}+\left(\dot{\epsilon}_{\mathbf{H}}, \mathcal{P} \phi\right)_{W} \\
& =\left(\dot{\epsilon}_{\mathbf{H}}, u\right)_{W}+\left(\mathcal{D} \dot{\epsilon}_{\mathbf{H}}, \phi\right) \\
& =\left(\dot{\epsilon}_{\mathbf{H}}, u\right)_{W} .
\end{aligned}
$$

The last step follows from (4.8). Lemma 4 follows by a scale change argument for $u$ as in the estimate (6.3).

Proof of Lemma 5. The proof is similar to that of Lemma 4. We note that the quadrature rule (see Figure 2)

$$
\int_{\kappa_{j}^{\prime}} f(y, z) d \sigma \approx f(Q) s_{j}^{\prime}
$$

is exact for constant functions $f$ when the point $Q$ is not the center of the rectangle $O^{\prime} O B A$. By a Taylor expansion we can show that after adding the following correction term

$$
\frac{1}{2}\left[f_{y}\left(P_{2}\right){\overline{P_{2} Q}}^{2}-f_{y}\left(P_{1}\right){\overline{P_{1} Q}}^{2}\right]{\overline{P_{3} P_{4}}}+\frac{1}{2}\left[f_{z}\left(P_{4}\right){\overline{P_{4} Q}}^{2}-f_{z}\left(P_{3}\right){\overline{P_{3} Q}}^{2}\right] \overline{P_{1} P_{2}}
$$

the quadrature rule is exact for linear polynomials $f(y, z)$. So on co-face $\kappa_{j}^{\prime}$, we split $\left(\delta_{\mathbf{E}}\right)_{j}$ as

$$
s_{j}^{\prime}\left(\delta_{\mathbf{E}}\right)_{j}=s_{j}^{\prime} v_{j}^{1}+v_{j}^{3}
$$


where $v_{j}^{3}:=\left(C^{\prime} v_{j}^{2}\right)_{\kappa_{j}^{\prime}}$, i.e., $v_{j}^{3}$ is expressed as a discrete curl. Using $P_{i}, i=1, \cdots, 4$, Figure 2 shows how this discrete curl is formed in terms of $v_{j}^{2}$ :

$$
v_{j}^{2}\left(P_{1}\right):=\frac{1}{2}\left(h_{x}^{2} \mathbf{E}_{2 x}\left(P_{1}\right)+h_{y}^{2} \mathbf{E}_{1 y}\left(P_{1}\right)\right)
$$

and $v_{j}^{1}$ is computed from (6.8) and vanishes for piecewise linear polynomial fields E. By summation by parts

$$
\begin{aligned}
\left(\dot{\delta}_{\mathbf{E}}, \eta_{\mathbf{E}}\right)_{W^{\prime}} & =\left(\dot{v}^{1}, \eta_{\mathbf{E}}\right)_{W^{\prime}}+\left(C^{\prime} \dot{v}^{2}, D \eta_{\mathbf{E}}\right) & & \text { by }(3.3) \\
& =\left(\dot{v}^{1}, \eta_{\mathbf{E}}\right)_{W^{\prime}}+\left(D^{\prime} \dot{v}^{2}, C \eta_{\mathbf{E}}\right) & & \text { by Lemma } 1 \\
& =\left(\dot{v}^{1}, \eta_{\mathbf{E}}\right)_{W^{\prime}}+\mu\left(D^{\prime} \dot{v}^{2}, S \dot{\epsilon}_{\mathbf{H}}\right) & & \text { by }(4.6) \\
& =\left(\dot{v}^{1}, \eta_{\mathbf{E}}\right)_{W^{\prime}}+\mu\left(\dot{v}^{2}, \dot{\epsilon}_{\mathbf{H}}\right)_{W} & & \text { by }(3.1),
\end{aligned}
$$

and the estimate (6.5) follows from (6.9) and the fact that the continuous linear functional $v_{i}^{1}$ vanishes for linear polynomials $\mathbf{E}$.

\section{REFERENCES}

[1] Philippe G. Ciarlet, The Finite Element Method For Elliptic Problems, North-Holland Publishing Company, 1978. MR 58:25001

[2] G.Duvaut and J. Lions, Inequalities in Mechanics and Physics, Springer-Verlag, New York, 1976. MR 58:25191

[3] R. Holland, Finite-difference solution of Maxwell's equations in generalized nonorthogonal coordinates, IEEE Trans. Nuclear Science, Vol. NS-30, 1983, pp. 4589-4591.

[4] T.G. Jurgens, A. Taflove, K.Umashankar and T.G. Moore, Finite-difference timedomain modeling of curved surfaces, IEEE Trans. Antennas and Propagation, Vol. 40, 1992, pp. 1703-1708.

[5] T.G. Jurgens and A. TAflove, Three-dimensional contour FDTD modeling of scattering from single and multiple bodies, IEEE Trans. Antennas and Propagation, Vol. 41, 1993, pp. $1429-1438$

[6] J.-F. LEE, Numerical solutions of TM scattering using obliquely Cartesian finite difference time domain algorithm, IEEE Proc. H (Microwaves, Antennas and Propagation), Vol. 140, 1992, pp. 23-28.

[7] N. MadSEn, Divergence preserving discrete surface integral methods for Maxwell's equations using nonorthogonal unstructured grids, J. of Computational Physics, 119, 1995, pp. 34-45. MR 96b:78002

[8] N. Madsen and R.W. Ziolkowski, Numerical solution of Maxwell's equations in the time domain using irregular nonorthogonal grids, Wave Motion 10, 1988, pp. 583-596. MR 89j:78004

[9] N. Madsen and R.W. Ziolkowski, A three dimensional modified finite volume technique for Maxwell's equations, Electromagnetics, Vol. 10, 1990, pp. 147-161.

[10] B.J. McCartin and J.F. Dicello, Three dimensional finite difference frequency domain scattering computation using the Control Region Approximation, IEEE Trans. Magnetics, Vol. 25, No. 4, 1989, pp. 3092-3094.

[11] Peter Monk and Endre Suli, A Convergence Analysis of Yee's Scheme on Nonuniform Grids, SIAM J. of Numerical Anal. Vol 31, 1994, pp. 393-412. MR 95a:65191

[12] Peter Monk and Endre Suli, Error estimates for Yee's method on nonuniform grids, IEEE Trans. Magnetics, Vol 30, 1994, pp. 3200-3203.

[13] R.A. Nicolaides, Direct Discretization of Planar Div-Curl Problems, SIAM J. Numerical Anal. Vol 29, pp. 32-56 (1992). MR 93b:65176

[14] R.A. NicOlaides, Flow discretization by complementary volume techniques, AIAA paper 89-1978, Proceedings of 9th AIAA CFD Meeting, Buffalo, NY, June 1989.

[15] R.A. Nicolaides, Analysis and convergence of the MAC scheme. 1. The linear problem, SIAM J. Numerical Anal., Vol 29, No. 6, pp. 1579-1591, 1992. MR 93j:65143

[16] R.A. Nicolaides and X.Wu, Analysis and convergence of the MAC scheme. 2. Navier-Stokes equations. Math. Comp. Vol. 65, No. 213, 29-44 (1996). MR 96d:65148.

[17] R.A. Nicolaides and X.Wu, Covolume solutions of three dimensional div-curl equations, SIAM J. Numer. Anal. Vol. 34, No. 6, pp. 2195-2203 (1997). CMP 98:04 
[18] K. YeE, Numerical Solution of Initial Boundary Value Problems Involving Maxwell's Equations in Isotropic Media, IEEE Trans. Antennas and Propagation, AP-16 (1966) pp. 302-307.

Department of Mathematics, Carnegie Mellon University, Pittsburgh, PA 15213

Current address: Department of Mathematics, Carnegie Mellon University, Pittsburgh, PA 15213

E-mail address: rnOm@andrew.cmu.edu

Department of Mathematics, Carnegie Mellon University, Pittsburgh, PA 15213

Current address: Department of Mathematical Sciences, University of Delaware, Newark, DE 19716

E-mail address: dqwang@math.udel.edu 